\title{
A $q$-ANALOGUE OF THE FKG INEQUALITY AND SOME APPLICATIONS
}

\author{
ANDERS BJÖRNER
}

Abstract. Let $L$ be a finite distributive lattice and $\mu: L \rightarrow \mathbb{R}^{+}$a logsupermodular function. For functions $k: L \rightarrow \mathbb{R}^{+}$let

$$
E_{\mu}(k ; q) \stackrel{\text { def }}{=} \sum_{x \in L} k(x) \mu(x) q^{\mathrm{rank}(x)} \in \mathbb{R}^{+}[q] .
$$

We prove for any pair $g, h: L \rightarrow \mathbb{R}^{+}$of monotonely increasing functions, that

$$
E_{\mu}(g ; q) \cdot E_{\mu}(h ; q) \ll E_{\mu}(1 ; q) \cdot E_{\mu}(g h ; q),
$$

where "«" denotes coefficientwise inequality of real polynomials. The FKG inequality of Fortuin, Kasteleyn and Ginibre (1971) is the real number inequality obtained by specializing to $q=1$.

The polynomial FKG inequality has applications to $f$-vectors of joins and intersections of simplicial complexes, to Betti numbers of intersections of certain Schubert varieties, and to the following kind of correlation inequality for power series weighted by Young tableaux.

Let $Y$ be the set of all integer partitions. Given functions $k, \mu: Y \rightarrow \mathbb{R}^{+}$, and parameters $0 \leq s \leq t$, define the formal power series

$$
F_{\mu}(k ; z) \stackrel{\text { def }}{=} \sum_{\lambda \in Y} k(\lambda) \mu(\lambda)\left(f_{\lambda}\right)^{t} \frac{z^{|\lambda|}}{(|\lambda| !)^{s}} \in \mathbb{R}^{+}[[z]],
$$

where $f_{\lambda}$ is the number of standard Young tableaux of shape $\lambda$. Assume that $\mu: Y \rightarrow \mathbb{R}^{+}$is log-supermodular, and that $g, h: Y \rightarrow \mathbb{R}^{+}$are monotonely increasing with respect to containment order of partition shapes. Then

$$
F_{\mu}(g ; z) \cdot F_{\mu}(h ; z) \ll F_{\mu}(1 ; z) \cdot F_{\mu}(g h ; z) .
$$

\section{INTRODUCTION}

Suppose that $L$ is a finite lattice, and for functions $k, \mu: L \rightarrow \mathbb{R}$ let

$$
E_{\mu}[k] \stackrel{\text { def }}{=} \sum_{x \in L} k(x) \mu(x) .
$$

A function $g: L \rightarrow \mathbb{R}$ is increasing if $x \leq y$ implies $g(x) \leq g(y)$, and decreasing if $x \leq y$ implies $g(x) \geq g(y)$. Two functions $g, h: L \rightarrow \mathbb{R}$ are comonotone if either both are increasing or else both are decreasing. They are countermonotone if one is increasing and the other decreasing.

2000 Mathematics Subject Classification. 05A20; 05E10; 60C05.

Research supported by the Knut and Alice Wallenberg Foundation, grant KAW.2005.0098. 
Let $\mathbb{R}^{+} \stackrel{\text { def }}{=}\{r \in \mathbb{R}: r \geq 0\}$. A function $\mu: L \rightarrow \mathbb{R}^{+}$is said to be logsupermodular if

$$
\mu(x) \mu(y) \leq \mu(x \wedge y) \mu(x \vee y), \text { for all } x, y \in L .
$$

The following theorem is due to Fortuin, Kasteleyn and Ginibre [8].

Theorem 1.1. (FKG inequality) Let $L$ be a finite distributive lattice, $\mu: L \rightarrow \mathbb{R}^{+}$ a log-supermodular weight function, and $g$ and $h$ comonotone functions $g, h: L \rightarrow$ $\mathbb{R}^{+}$. Then

$$
E_{\mu}[g] \cdot E_{\mu}[h] \leq E_{\mu}[1] \cdot E_{\mu}[g h]
$$

For countermonotone functions the inequality is reversed.

The FKG inequality arose as a correlation inequality in statistical mechanics, more precisely in the study of Ising ferromagnets and the random cluster model. It has found many applications in extremal and probabilistic combinatorics. See e.g. [2] for an expository account and pointers to related results.

In this paper we prove a polynomial inequality that implies the original FKG inequality via specialization, and which provides more detailed information. The basic version is given in Section 2 and a more elaborate one in Section 5.

The polynomial FKG inequality has applications to $f$-vectors of joins and intersections of simplicial complexes and to Betti numbers of intersections of certain Schubert varieties. These applications appear in Sections 3 and 4.

In Section 6 we derive a class of correlation inequalities for power series on partitions weighted by Young tableaux. This class contains the poissonization of Plancherel measure, a probability measure on the set $Y$ of all integer partitions discussed in [5].

\section{A $q$-FKG INEQUALITY}

Only the most basic facts about distributive lattices are used in this paper. Standard facts and notation can be found in e.g. [4] or [13]. For instance, we use that a finite such lattice has bottom and top elements $\widehat{0}$ and $\widehat{1}$ and a rank function $r$, such that $r(x)$ equals the length of any maximal chain in the interval $[\widehat{0}, x]$. Furthermore, this function satisfies the modular law

$$
r(x)+r(y)=r(x \wedge y)+r(x \vee y), \quad \text { for all } x, y \in L .
$$

For polynomials $p(q), s(q) \in \mathbb{R}[q]$,

$$
\text { let } p(q) \ll s(q) \text { mean that } s(q)-p(q) \in \mathbb{R}^{+}[q] \text {. }
$$

Let $L$ be a finite distributive lattice with rank function $r$. For functions $\mu, k$ : $L \rightarrow \mathbb{R}^{+}$define the polynomial

$$
E_{\mu}(k ; q) \stackrel{\text { def }}{=} \sum_{x \in L} k(x) \mu(x) q^{r(x)} \in \mathbb{R}^{+}[q] .
$$

Theorem 2.1. Let $L$ be a finite distributive lattice, $\mu: L \rightarrow \mathbb{R}^{+}$a log-supermodular weight function and $g$ and $h$ comonotone functions $g, h: L \rightarrow \mathbb{R}^{+}$. Then

$$
E_{\mu}(g ; q) \cdot E_{\mu}(h ; q) \ll E_{\mu}(1 ; q) \cdot E_{\mu}(g h ; q) .
$$


For countermonotone functions the inequality is reversed.

The original FKG inequality (Theorem 1.1) is obtained by putting $q=1$. Slightly more general $q$-FKG inequalies are given in Theorem 5.3.

Proof. We may assume that both $g$ and $h$ are increasing functions on $L$. The cases when one or both are decreasing are easily deduced from this.

Let

$$
\Phi(q) \stackrel{\text { def }}{=} E_{\mu}(1 ; q) \cdot E_{\mu}(g h ; q)-E_{\mu}(g ; q) \cdot E_{\mu}(h ; q),
$$

and for $x, y \in L$ let

$$
\phi(x, y) \stackrel{\text { def }}{=} \mu(x) \mu(y)[g(x)-g(y)][h(x)-h(y)] .
$$

Note that $\phi(x, y)=\phi(y, x)$. A simple computation, consisting of grouping all the terms of $\Phi(q)$ that contain $\mu(x) \mu(y)$ together, shows that

$$
\Phi(q)=\sum_{\{x, y\} \in\left(\begin{array}{c}
L \\
2
\end{array}\right)} \phi(x, y) q^{r(x)+r(y)} .
$$

Hence, the degree $d$ coefficient of $\Phi(q)$ equals

$$
\Phi_{d}=\sum_{\{x, y\} \in\left(\begin{array}{l}
L \\
2
\end{array}\right), r(x)+r(y)=d} \phi(x, y) .
$$

Now consider another, slightly coarser, grouping of terms. For $u, v \in L$ such that $u \leq v$, let $C(u, v)$ denote the set of all pairs of relative complements in the interval $[u, v]$, that is, unordered pairs $\{x, y\}$ such that $x \wedge y=u, x \vee y=v$. Let

$$
\psi(u, v) \stackrel{\text { def }}{=} \sum_{\{x, y\} \in C(u, v)} \phi(x, y) .
$$

It follows from equation (2.2) and the modular law (2.1) for the rank function that

$$
\Phi_{d}=\sum_{u \leq v, r(u)+r(v)=d} \psi(u, v) .
$$

Our aim is to prove the following.

Claim. For all $u \leq v$ in $L$

$$
\psi(u, v) \geq 0 .
$$

The nonegativity of $\Phi_{d}$ then follows.

To prove Claim (2.5) we may without loss of generality make the following three assumptions:

(i) $\mu(x)>0$ for all $x \in L$,

(ii) $u=\widehat{0}$ and $v=\widehat{1}$,

(iii) $L$ is Boolean. 
The reasons that these simplifications are valid are as follows.

(i) Let $L^{\prime} \stackrel{\text { def }}{=}\{x \in L: \mu(x)>0\}$. If $x \in L^{\prime}$ and $y \in L^{\prime}$ then log-supermodularity implies that also $x \wedge y \in L^{\prime}$ and $x \vee y \in L^{\prime}$. Hence, $L^{\prime}$ is a sublattice. Since elements outside $L^{\prime}$ don't contribute to the sums involved in the statement of the theorem we may pass from $L$ to consideration of the distributive sublattice $L^{\prime}$.

(ii) Every interval $[u, v]$ is itself a finite distributive lattice, and what goes into Claim (2.5) depends only on this substructure.

(iii) It is known that the set $C(\widehat{0}, \widehat{1})$ of complemented elements in a finite distributive lattice $L$ forms a Boolean sublattice, see [4, p. 18]. By definition (2.3), $\psi(\widehat{0}, \widehat{1})$ only depends on the restriction to this Boolean sublattice.

After these simplifications we return to the proof of Claim (2.5), for which it remains to show that

$$
\psi(\widehat{0}, \widehat{1}) \geq 0
$$

in a finite Boolean lattice $L$. This will be done by induction on the rank of $L$. The inequality is clearly valid for $\operatorname{rank}(L)=1$, in which case $L=\{\widehat{0}, \widehat{1}\}$.

Suppose then that $L$ is Boolean, and for each element $x \in L$, let $x^{c}$ denote its complement in $L$. Let $B \stackrel{\text { def }}{=}\left[\widehat{0}, a^{c}\right]$ for some atom (rank one element) $a \in L$.

Let $\widetilde{\mu}(x) \stackrel{\text { def }}{=} \mu(x) \mu\left(x^{c}\right)$. Note that $\widetilde{\mu}$ is a log-supermodular function on $L$, and hence in particular on $B$, and that

$$
\sum_{x \in B} \widetilde{\mu}(x)=\sum_{x \in B} \widetilde{\mu}\left(x^{c}\right)=\sum_{x \in B} \widetilde{\mu}(x \vee a) .
$$

Let

$$
\alpha \stackrel{\text { def }}{=} \sum_{x \in B}\left[g(x)-g\left(x^{c}\right)\right] \widetilde{\mu}(x) \quad \text { and } \quad \beta \stackrel{\text { def }}{=} \sum_{x \in B}\left[h(x)-h\left(x^{c}\right)\right] \widetilde{\mu}(x) .
$$

The plan is to show that

$$
\psi(\widehat{0}, \widehat{1}) \geq \alpha \cdot \beta
$$

and

$$
\alpha \leq 0 \text { and } \beta \leq 0
$$

from which inequality (2.6) follows.

The interval $B$ is a Boolean lattice of rank one less than $L$. Hence, by the induction hypothesis $\psi(u, v) \geq 0$ for all $u \leq v$ in $B$, which means that the statement of the theorem is valid for $B$. This statement, specialized to $q=1$, shows that for any pair of increasing nonnegative functions $s, t$ on $B$ :

$$
\sum_{x \in B} s(x) \widetilde{\mu}(x) \cdot \sum_{x \in B} t(x) \widetilde{\mu}(x) \leq \sum_{x \in B} \widetilde{\mu}(x) \cdot \sum_{x \in B} s(x) t(x) \widetilde{\mu}(x) .
$$

Now, define two functions $B \rightarrow \mathbb{R}^{+}$this way:

$$
\begin{gathered}
\widetilde{g}(x) \stackrel{\text { def }}{=} e+g(x)-g\left(x^{c}\right) \\
\widetilde{h}(x) \stackrel{\text { def }}{=} e+h(x)-h\left(x^{c}\right),
\end{gathered}
$$


where $e$ is some number such that $e \geq g(\widehat{1}), h(\widehat{1})$. Then $\widetilde{g}$ and $\widetilde{h}$ are nonnegative and increasing. Hence, by the induction hypothesis $(2.10)$

$$
\sum_{x \in B} \widetilde{g}(x) \widetilde{\mu}(x) \cdot \sum_{x \in B} \widetilde{h}(x) \widetilde{\mu}(x) \leq \sum_{x \in B} \widetilde{\mu}(x) \cdot \sum_{x \in B} \widetilde{g}(x) \widetilde{h}(x) \widetilde{\mu}(x) .
$$

After expanding these products and cancelling terms this inequality reduces to

$$
\begin{gathered}
\sum_{x \in B}\left[g(x)-g\left(x^{c}\right)\right] \widetilde{\mu}(x) \cdot \sum_{x \in B}\left[h(x)-h\left(x^{c}\right)\right] \widetilde{\mu}(x) \\
\leq \sum_{x \in B}\left[g(x)-g\left(x^{c}\right)\right]\left[h(x)-h\left(x^{c}\right)\right] \widetilde{\mu}(x),
\end{gathered}
$$

which is inequality $(2.8)$

Next, let

$$
\gamma(x) \stackrel{\text { def }}{=} \frac{\widetilde{\mu}(x \vee a)}{\widetilde{\mu}(x)} .
$$

Since $\widetilde{\mu}(x)>0$ this defines a function $\gamma: B \rightarrow \mathbb{R}^{+}$, which due to the logsupermodularity of $\widetilde{\mu}$ is increasing:

$$
x<y \text { and } y \in B \Rightarrow \widetilde{\mu}(y) \widetilde{\mu}(x \vee a) \leq \widetilde{\mu}(x) \widetilde{\mu}(y \vee a) \Rightarrow \gamma(x) \leq \gamma(y) .
$$

By the induction hypothesis (2.10)

$$
\sum_{x \in B} g(x) \widetilde{\mu}(x) \cdot \sum_{x \in B} \gamma(x) \widetilde{\mu}(x) \leq \sum_{x \in B} \widetilde{\mu}(x) \cdot \sum_{x \in B} g(x) \gamma(x) \widetilde{\mu}(x),
$$

which because of equation (2.7) reduces to

$$
\sum_{x \in B} g(x) \widetilde{\mu}(x) \leq \sum_{x \in B} g(x) \widetilde{\mu}(x \vee a) .
$$

This together with

$$
\sum_{x \in B} g(x) \widetilde{\mu}(x \vee a) \leq \sum_{x \in B} g(x \vee a) \widetilde{\mu}(x \vee a)=\sum_{x \in B} g\left(x^{c}\right) \widetilde{\mu}\left(x^{c}\right)=\sum_{x \in B} g\left(x^{c}\right) \widetilde{\mu}(x)
$$

proves inequality (2.9) for $\alpha$. The analogous argument for $\beta$ then finishes the proof.

\section{FACE NUMBers of INTERSECTIONS OF SimpliCiAL COMPLEXES}

Let $\Delta$ and $\Gamma$ be simplicial complexes on the same finite vertex set $V$. Define the $f$-polynomial $f_{\Delta}(q)=\sum_{i>0} f_{i-1}(\Delta) q^{i}$, where $f_{i-1}(\Delta)$ denotes the number of $(i-1)$-dimensional faces, and similarly for $\Gamma$.

\section{Theorem 3.1.}

$$
f_{\Delta}(q) \cdot f_{\Gamma}(q) \ll(1+q)^{|V|} \cdot f_{\Delta \cap \Gamma}(q)
$$

Proof. Apply Theorem 2.1 to the Boolean lattice $2^{V}$, putting

$$
\mu(x) \equiv 1, \quad g(x)=\left\{\begin{array}{l}
1, \text { if } x \in \Delta \\
0, \text { if } x \notin \Delta
\end{array} \quad \text {, and } \quad h(x)=\left\{\begin{array}{l}
1, \text { if } x \in \Gamma \\
0, \text { if } x \notin \Gamma
\end{array}\right.\right.
$$


The theorem can equivalently be formulated in terms of the simplicial join operation:

$$
f_{i}(\Delta * \Gamma) \leq f_{i}\left(2^{V} *(\Delta \cap \Gamma)\right), \text { for all } i
$$

For $q=1$ the theorem specializies to a result of Kleitman [10].

\section{Poincaré polynomials for intersections of Schubert VARIETiEs}

Let $G$ be a complex semisimple algebraic group and $P$ a maximal parabolic subgroup. In the homogeneous space $G / P$, known as a generalized Grassmannian, there are Schubert varieties $X_{u}$, indexed by elements $u \in W^{J}$. Here $(W, S)$ is the Weyl group of $G, J=S \backslash\left\{s_{\alpha}\right\}$, and $W^{J}$ the Bruhat poset of minimal coset representatives modulo the corresponding maximal parabolic subgroup $W_{J}$ of $W$, obtained by removing the node corresponding to $\alpha$ from the Coxeter-Dynkin diagram of $G$.

We refer to the literature, e.g. [3] and [9], for definitions and explanations of these and other notions concerning algebraic groups, root systems and Schubert varieties. For instance, we consider the notion of minuscule weight known, see [3, p. 21] or [9, pp. 172-174]. The Grassmannian $G / P$ is of minuscule type if the fundamental weight $\alpha$ is minuscule. The Grassmannians of minuscule type have been classified. In addition to the classical Grassmannians of $k$-dimensional subspaces in $\mathbb{C}^{d}$ (the type $A$ case), there are four infinite families and three sporadic cases.

The Poincaré polynomial of a Schubert variety $X_{u}$ is $P_{X_{u}}(q) \stackrel{\text { def }}{=} \sum \beta_{i}\left(X_{u}\right) q^{i}$, where the Betti numbers are the dimensions of classical singular cohomology groups with complex coefficients: $\beta_{i}\left(X_{u}\right)=\operatorname{dim} H^{i}\left(X_{u} ; \mathbb{C}\right)$. Explicit expressions for $P_{G / P}(q)$ are known in terms of parameters of the groups in all cases.

Theorem 4.1. Let $X_{u}$ and $X_{v}$ be Schubert varieties in a Grassmannian $G / P$ of minuscule type. Then,

$$
P_{X_{u}}(q) \cdot P_{X_{v}}(q) \ll P_{G / P}(q) \cdot P_{X_{u} \cap X_{v}}(q)
$$

Proof. It is a well-known consequence of the decomposition into Schubert cells that $\beta_{2 i}\left(X_{u}\right)$ equals the number of elements $w$ such that $\ell(w)=i$ and $w \leq u$ in the Bruhat poset $W^{J}$. Also, $\beta_{2 i+1}\left(X_{u}\right)=0$. On the other hand, it is known from the work of Proctor [11] that $W^{J}$ is a distributive lattice if and only if $J=S \backslash\left\{s_{\alpha}\right\}$ for a minuscule weight $\alpha$. Being a lattice implies that $X_{u} \cap X_{v}=X_{u \wedge v}$.

Hence, the result is obtained by applying Theorem 2.1 to $W^{J}$, letting

$$
\mu(w) \equiv 1, \quad g(w)=\left\{\begin{array}{l}
1, \text { if } w \leq u \\
0, \text { if } w \leq \leq u
\end{array} \quad \text { and } \quad h(w)=\left\{\begin{array}{l}
1, \text { if } w \leq v \\
0, \text { if } w \leq \leq v
\end{array} .\right.\right.
$$

\section{A more general $q$-FKG INEQUALity}

Let $L$ be a finite distributive lattice, and for $x \in L$ let $m(x)$ denote the number of maximal chains in the interval $[\widehat{0}, x]$. The following result is due to Fishburn 
[7] in an equivalent version formulated for linear extensions of posets, see also [6, Lemma 3.1].

Lemma 5.1. (Fishburn) The function $x \mapsto \frac{m(x)}{r(x) !}$ is log-supermodular on $L$.

We need is a slightly elaborated version.

Proposition 5.2. Let $0 \leq s \leq t$ be real numbers. Then the function $x \mapsto \frac{m^{t}(x)}{(r(x) !)^{s}}$ is log-supermodular on $L$.

Proof. It follows from the modular law for the rank function (2.1) that $x \mapsto$ $r(x)$ ! is log-supermodular. Also, log-supermodularity is preserved by powers and products. Fishburn's lemma and

$$
\frac{m^{t}(x)}{(r(x) !)^{s}}=\left(\frac{m(x)}{r(x) !}\right)^{t} \cdot(r(x) !)^{t-s}
$$

therefore complete the proof.

Theorem 5.3. Let $L$ be a finite distributive lattice, $\mu: L \rightarrow \mathbb{R}^{+}$a log-supermodular weight function, and $0 \leq s \leq t$. For functions $k: L \rightarrow \mathbb{R}^{+}$let

$$
E(k ; q) \stackrel{\text { def }}{=} \sum_{x \in L} k(x) \mu(x) m^{t}(x) \frac{q^{r(x)}}{(r(x) !)^{s}} \in \mathbb{R}^{+}[q] .
$$

If $g, h: L \rightarrow \mathbb{R}^{+}$are comonotone functions, then

$$
E(g ; q) \cdot E(h ; q) \ll E(1 ; q) \cdot E(g h ; q) .
$$

For countermonotone functions the inequality is reversed.

Proof. The inequality follows from Theorem 2.1 and Proposition 5.2.

\section{Series Weighted By Young tableaux}

Young's lattice $Y$ consists of all number partitions ordered by inclusion of their Ferrers diagrams. It is a distributive lattice. For two partitions

$$
\lambda=\left(\lambda_{1} \geq \lambda_{2} \geq \cdots\right) \quad \text { and } \quad \sigma=\left(\sigma_{1} \geq \sigma_{2} \geq \cdots\right)
$$

the lattice operations are

$$
\lambda \vee \sigma=\left(\max \left(\lambda_{1}, \sigma_{1}\right) \geq \max \left(\lambda_{2}, \sigma_{2}\right) \geq \cdots\right)
$$

and

$$
\lambda \wedge \sigma=\left(\min \left(\lambda_{1}, \sigma_{1}\right) \geq \min \left(\lambda_{2}, \sigma_{2}\right) \geq \cdots\right) .
$$

For notation and definitions concerning number partitions, Young tableaux and Young's lattice we refer to $\left[14, \mathrm{Ch}\right.$. 7]. In particular, $f_{\lambda}$ denotes the number of standard Young tableaux of partition shape $\lambda$, and $|\lambda|=\sum \lambda_{i}$. 
Proposition 6.1. Suppose that $0 \leq s \leq t$. Then the function

$$
\lambda \mapsto \frac{f_{\lambda}^{t}}{(|\lambda| !)^{s}}
$$

from $Y$ to $\mathbb{R}^{+}$is log-supermodular.

Proposition 6.1 is the Young's lattice special case of Proposition 5.2 and therefore relies on Lemma 5.1. Since the proofs in [7] and [6] for Lemma 5.1 are somewhat involved, we provide a simpler direct proof for the Young's lattice special case, thus making this paper self-contained concerning the results in this section. Our proof is based on the hook formula and Lemma 6.2.

Let $L$ be a distributive lattice, and for $x, y \in L$ let $d(x, y) \stackrel{\text { def }}{=} r(x \vee y)-r(x \wedge y)$. Then $d$ is a metric on $L$ (we don't use this fact), and $d(x, y)=2$ if and only if $x$ and $y$ are either the top and bottom elements or the two middle elements of an interval of length 2 .

Lemma 6.2. A function $\mu: L \rightarrow \mathbb{R}^{+} \backslash\{0\}$ is log-supermodular if and only if

$$
\mu(x) \mu(y) \leq \mu(x \wedge y) \mu(x \vee y) \text { for all } x, y \in L \text { such that } d(x, y)=2 .
$$

Proof. Suppose that the inequality holds for all $x, y$ such that $d(x, y)=2$. We prove that it then holds for all pairs $x, y$, by induction on $d(x, y)$.

Suppose that $d(x, y) \geq 3$ and (without loss of generality) that $r(x)-r(x \wedge y) \geq$ 2. Let $a$ be an atom in the interval $[x \wedge y, x]$. Notice that $d(a, y)<d(x, y)$ and $d(x, a \vee y)<d(x, y)$. Hence, the induction assumption gives

$$
\frac{\mu(y)}{\mu(x \wedge y)}=\frac{\mu(y)}{\mu(a \wedge y)} \leq \frac{\mu(a \vee y)}{\mu(a)} \leq \frac{\mu(x \vee y)}{\mu(x)}
$$

Proof of the Young's lattice case of Lemma 5.1.

For two partitions $\lambda$ and $\sigma$, let $\sigma \backslash \lambda$ denote the set of cells in the Ferrers diagram of $\sigma$ that are not in $\lambda$. By Lemma 6.2 we may in the following assume that $|\sigma \backslash \lambda|=1$ and that $|\lambda \backslash \sigma|=1$. Let $s \stackrel{\text { def }}{=}|\sigma|-1=|\lambda|-1$.

Let $c$ be the cell of the Ferrers diagram defined by $\sigma \backslash \lambda=\{c\}$. Then,

$$
\lambda \wedge \sigma=\sigma \backslash c \text { and } \lambda \vee \sigma=\lambda \cup c .
$$

Using the hook formula for $f_{\lambda}$ (see $[14, \mathrm{p} . \mathrm{xx}]$ ), the inequality we have to prove is the following

$$
\frac{1}{\prod_{a \in \lambda} h_{a}} \cdot \frac{1}{\prod_{b \in \sigma} h_{b}} \leq \frac{1}{\prod_{a \in \lambda \cup c} h_{a}} \cdot \frac{1}{\prod_{b \in \sigma \backslash c} h_{b}},
$$

which we rewrite

$$
\prod_{a \in \lambda} h_{a}^{\lambda} \prod_{b \in \sigma} h_{b}^{\sigma} \geq \prod_{a \in \lambda \cup c} h_{a}^{\lambda \cup c} \prod_{b \in \sigma \backslash c} h_{b}^{\sigma \backslash c}
$$

where the upper indices of the hook numbers refer to the respective shapes in question. 
Let $Z$ be the set of all cells of the Ferrers diagram, other than $c$, whose place is in the same row or same column as the critical cell $c$. We observe that

$$
h_{a}^{\lambda}=h_{a}^{\lambda \cup c} \text { and } h_{b}^{\sigma}=h_{b}^{\sigma \backslash c} \text { for all cells } a, b \text { not in } Z,
$$

and

$$
h_{a}^{\lambda \cup c}=h_{a}^{\lambda}+1 \text { and } h_{b}^{\sigma \backslash c}=h_{b}^{\sigma}-1 \text { for all cells } a, b \in Z .
$$

Thus, cancelling equal factors in inequality (6.1) all that remains to prove is that

$$
\prod_{a \in Z} h_{a}^{\lambda} \prod_{b \in Z} h_{b}^{\sigma} \geq \prod_{a \in Z}\left(h_{a}^{\lambda}+1\right) \prod_{b \in Z}\left(h_{b}^{\sigma}-1\right) .
$$

This is clear since $\sigma \backslash c \subseteq \lambda$ implies that $h_{a}^{\sigma} \leq h_{a}^{\lambda}$, and hence

$$
h_{a}^{\lambda} h_{a}^{\sigma} \geq\left(h_{a}^{\lambda}+1\right)\left(h_{a}^{\sigma}-1\right), \text { for all } a \in Z \text {. }
$$

For formal power series $G(z), H(z) \in \mathbb{R}[[z]]$,

$$
\text { let } G(z) \ll H(z) \text { mean that } H(z)-G(z) \in \mathbb{R}^{+}[[z]] \text {. }
$$

We obtain the following correlation-type inequality for monotone functions on Young's lattice.

Theorem 6.3. Suppose that $\mu: Y \rightarrow \mathbb{R}^{+}$is log-supermodular and $0 \leq s \leq t$. For functions $k: Y \rightarrow \mathbb{R}^{+}$let

$$
F(k ; z) \stackrel{\text { def }}{=} \sum_{\lambda \in Y} k(\lambda) \mu(\lambda)\left(f_{\lambda}\right)^{t} \frac{z^{|\lambda|}}{(|\lambda| !)^{s}} \in \mathbb{R}^{+}[[z]] .
$$

If $g, h: Y \rightarrow \mathbb{R}^{+}$are comonotone functions, then

$$
F(g ; z) \cdot F(h ; z) \ll F(1 ; z) \cdot F(g h ; z) .
$$

For countermonotone functions the inequality is reversed.

Proof. To prove this coefficient-wise inequality for the degree $d$ term it suffices to consider the truncations of the four power series to polynomials of degree $d$, obtained by omitting all higher-degree terms. Consider the partition $d^{d} \stackrel{\text { def }}{=}$ $(d, d, \ldots, d)$, and let $L \stackrel{\text { def }}{=}\left[\emptyset, d^{d}\right]$. Then $L$ is an interval in $Y$ and hence a finite distributive lattice. Now observe that the degree $d$ truncation of the polynomial $E(g ; z)$ of Theorem 5.3 computed on $L$ equals the degree $d$ truncation of $F(g ; z)$, and similarly for the other four factors. Hence, the result is a consequence of Theorem 5.3.

A special case deserves mention. The function

$$
\lambda \mapsto \frac{f_{\lambda}^{2}}{|\lambda| !}
$$

from $Y$ to $\mathbb{R}^{+}$is known as Plancherel measure on partitions, and

$$
\lambda \mapsto \pi_{\theta}(\lambda) \stackrel{\text { def }}{=} e^{-\theta} \frac{\theta^{|\lambda|} f_{\lambda}^{2}}{(|\lambda| !)^{2}}
$$

as its poissonization, see [5]. Here $\theta>0$ is a real parameter. 
Corollary 6.4. For functions $k: Y \rightarrow \mathbb{R}^{+}$let

$$
P(k ; z) \stackrel{\text { def }}{=} \sum_{\lambda \in Y} k(\lambda) \pi_{\theta}(\lambda) z^{|\lambda|}
$$

where $\pi_{\theta}$ is the poissonization of Plancherel measure. If $g, h: Y \rightarrow \mathbb{R}^{+}$are comonotone functions, then

$$
P(g ; z) \cdot P(h ; z) \ll P(1 ; z) \cdot P(g h ; z) .
$$

In particular, under condition of convergence there is the correlation inequality

$$
\sum_{\lambda \in Y} g(\lambda) \pi_{\theta}(\lambda) \cdot \sum_{\lambda \in Y} h(\lambda) \pi_{\theta}(\lambda) \leq \sum_{\lambda \in Y} g(\lambda) h(\lambda) \pi_{\theta}(\lambda)
$$

We end with a curiosity. Let $\lambda \vdash n$ denote that $\lambda$ is a partition of the integer $n$, let $p(n)$ be the number of partitions of $n$, and let $i(n)$ be the number of involutions of an $n$-element set. It is known that

$$
\sum_{\lambda \vdash n} f_{\lambda}^{2}=n !, \quad \sum_{\lambda \vdash n} f_{\lambda}=i(n), \quad \sum_{n \geq 0} i(n) \frac{z^{n}}{n !}=e^{z+z^{2} / 2},
$$

see [14]. Thus we have:

$$
\sum_{\lambda \in Y} f_{\lambda}^{k} \frac{z^{|\lambda|}}{|\lambda| !}=\left\{\begin{array}{l}
\sum_{n \geq 0} p(n) \frac{z^{n}}{n !}, \text { if } k=0 \\
e^{z+z^{2} / 2}, \text { if } k=1 \\
\frac{1}{1-z}, \text { if } k=2 .
\end{array}\right.
$$

Such explicit expressions do not seem to be known for other values of the power $k$. However, the following relations between such series can be observed.

The function $\lambda \mapsto f_{\lambda}^{s}$ is decreasing if $s<0$, and increasing if $s>0$. Hence, if $s t>0$ we obtain this special case of Theorem 6.3 :

$$
\left(\sum_{\lambda \in Y} \frac{f_{\lambda}^{s+1} z^{|\lambda|}}{|\lambda| !}\right)\left(\sum_{\lambda \in Y} \frac{f_{\lambda}^{t+1} z^{|\lambda|}}{|\lambda| !}\right) \ll e^{z+z^{2} / 2} \cdot \sum_{\lambda \in Y} \frac{f_{\lambda}^{s+t+1} z^{|\lambda|}}{|\lambda| !}
$$

whereas if $s t<0$ the inequality goes the other way.

\section{REMARKS}

1. The original FKG inequality has been generalized in several directions. For instance, Sahi [12] gives a polynomial version different from the one considered here.

Ahlswede-Daykin's four function theorem [1] states the following. Suppose that $L$ is a finite distributive lattice and that $\alpha, \beta, \gamma, \delta$ are nonnegative real functions on $L$ such that

$$
\alpha(x) \beta(y) \leq \gamma(x \vee y) \delta(x \wedge y)
$$


for all $x, y \in L$. Then

$$
\sum_{x \in \mathcal{A}} \alpha(x) \cdot \sum_{x \in \mathcal{B}} \beta(x) \leq \sum_{x \in \mathcal{A} \vee \mathcal{B}} \gamma(x) \cdot \sum_{x \in \mathcal{A} \wedge \mathcal{B}} \delta(x),
$$

for all subsets $\mathcal{A}, \mathcal{B} \subseteq L$. Here, $\mathcal{A} \vee \mathcal{B} \stackrel{\text { def }}{=}\{x \vee y: x \in \mathcal{A}, y \in \mathcal{B}\}$ and $\mathcal{A} \wedge \mathcal{B} \stackrel{\text { def }}{=}$ $\{x \wedge y: x \in \mathcal{A}, y \in \mathcal{B}\}$. The $\mathrm{FKG}$ inequality is obtained from this by putting

$$
\alpha=g \mu, \quad \beta=h \mu, \quad \gamma=g h \mu, \quad \delta=\mu \text { and } \mathcal{A}=\mathcal{B}=L .
$$

This prompts the following.

Question: Let $\alpha, \beta, \gamma, \delta: L \rightarrow \mathbb{R}^{+}$be functions as above, satisfying condition (7.1). Does it then follow that

$$
\sum_{x \in \mathcal{A}} \alpha(x) q^{r(x)} \cdot \sum_{x \in \mathcal{B}} \beta(x) q^{r(x)} \ll \sum_{x \in \mathcal{A} \vee \mathcal{B}} \gamma(x) q^{r(x)} \cdot \sum_{x \in \mathcal{A} \wedge \mathcal{B}} \delta(x) q^{r(x)} ?
$$

2. We are grateful to P. Brändén for bringing references [6] and [7] to our attention.

\section{REFERENCES}

[1] R. Ahlswede and D. E. Daykin, An inequality for the weights of two families of sets, their unions and intersections, Z. Wahrs. Verw. Gebiete 43 (1978), 183-185.

[2] N. Alon and J. H. Spencer, The Probabilistic Method, 3rd Ed., Wiley-Interscience, New York, 2008.

[3] S. Billey and V. Lakshmibai, Singular Loci of Schubert Varieties, Progress in Math. No. 182, Birkhäuser, Boston, 2000.

[4] G. Birkhoff, Lattice Theory, 3rd ed., Amer. Math. Soc. Colloquium Publ. No. 25, American Mathematical Society, Providence, RI, 1967.

[5] A. Borodin, A. Okounkov and G. Olshanski, Asymptotics of Plancherel measures for symmetric groups, J. Amer. Math. Soc. 13 (2000), 481-515.

[6] G. R. Brightwell and W. T. Trotter, A combinatorial approach to correlation inequalities, Discrete Math. 257 (2002), 311-327.

[7] P. C. Fishburn, A correlational inequality for linear extensions of a poset, Order 1 (1984), $127-137$.

[8] C. M. Fortuin, P. W. Kasteleyn, and J. Ginibre, Corrrelation inequalities on some partially ordered sets, Commun. Math. Phys. 22 (1971), 89-103.

[9] H. Hiller, Geometry of Coxeter Groups, Research Notes in Math. No. 54, Pitman, Boston, 1982.

[10] D. J. Kleitman, Families of non-disjoint subsets, J. Combinat. Theory 1 (1966), 153-155.

[11] R. A. Proctor, Bruhat lattices, plane partition generating functions, and minuscule representations, Europ. J. Combinatorics 5 (1984), 331-350.

[12] S. Sahi, The FKG inequality for partially ordered algebras, J. Theor. Probab. 21 (2008), 449-458

[13] R. P. Stanley, Enumerative Combinatorics, Vol. 1, Cambridge Studies in Advanced Mathemtics No. 49, Cambridge University Press, Cambridge, UK, 1997.

[14] R. P. Stanley, Enumerative Combinatorics, Vol. 2, Cambridge Studies in Advanced Mathemtics No. 62, Cambridge University Press, Cambridge, UK, 1999.

Institut Mittag-Leffler, Auravägen 17, S-182 60 Duursholm, Sweden

E-mail address: bjorner@mittag-leffler.se 
Kungl. Tekniska Högskolan, Matematiska Inst., S-100 44 Stockholm, Sweden

E-mail address: bjorner@math.kth.se 